\title{
Microgravity Isolation Control System Design via High-Order Sliding Mode Control
}

\section{Shkolnikov and Y. Shtessel}

Department of Electrical and Computer Engineering, University of Alabama in Huntsville and

M.Whorton and M. Jackson

TD55/Vehicle Control Systems, NASA/Marshall Space Flight Center

\begin{abstract}
Vibration isolation control system design for a microgravity experiment mount is considered. The controller design based on dynamic sliding manifold (DSM) technique is proposed to attenuate the accelerations transmitted to an isolated experiment mount either from a vibrating base or directly generated by the experiment, as well as to stabilize the internal dynamics of this nonminimum phase plant. An auxiliary DSM is employed to maintain the highorder sliding mode on the primary sliding manifold in the presence of uncertain actuator dynamics of second order. The primary DSM is designed for the closed-loop system in sliding mode to be a filter with given characteristics with respect to the input external disturbances.
\end{abstract}

\section{Introduction}

As a research laboratory, the International Space Station (ISS) provides the unique nearzero gravity environment of low-earth orbit for state-of-the-art $\mu \mathrm{g}$ science investigation [1]. However, due to a variety of vibro-acoustic disturbances on the ISS, the acceleration environment is expected to significantly exceed the requirements of many acceleration sensitive experiments. Thus, vibration isolation system is required to attenuate the anticipated disturbances to an acceptable level. The basic objective of a vibration isolation system is to attenuate the accelerations transmitted to an isolated experiment mount either from a vibrating base or from directly applied disturbances generated by the experiment. Passive isolation techniques are often adequate to provide sufficient attenuation of vibration disturbances in the high frequency regime, but isolation of low and intermediate frequency vibrations requires active isolation. 
The vibration isolation requirements for active isolation system design can be stated as follows [1]

1) The isolation system must directly transmit the very low frequency quasisteady accelerations below $0.01 \mathrm{~Hz}$.

2) The amount of attenuation between $0.01 \mathrm{~Hz}$ and $10 \mathrm{~Hz}$ must increase one order of magnitude for every decade of frequency.

3) Three orders of magnitude attenuation are required above $10 \mathrm{~Hz}$.

In order to provide a quiescent acceleration environment to an experiment, an active isolation system must sense and cancel the accelerations applied to the experiment. Standard approaches to microgravity vibration isolation typically employ high-frequency acceleration feedback control to cancel the accelerations and low frequency position feedback control to center the platform in the sway space while following the quasi-steady motion of the vehicle. Classical control approaches based on PID control and loop shaping have been fruitfully applied $\mu \mathrm{g}$ vibration isolation [1], while current work is also focusing on the development of more advanced multivariable linear control law development [2].

Microgravity vibration isolation systems must not only meet the performance requirements stated above, but they must also possess sufficient robustness to accommodate system properties such as significant cross-coupling, uncertain and possibly nonlinear umbilicals, varying mass and inertia properties, and uncertain flexible modes of the isolated payload. These issues of robustness and performance lead to a challenging control system design problem.

A robust Sliding Mode Control (SMC) design approach will be employed in this work to meet those requirements. For many control applications SMC has been proven [3] to be efficient technique to provide high-fidelity performance in stabilization or output-tracking problems for nonlinear systems with uncertainties in system parameters and external disturbances. The sliding mode is the system motion on a judiciously selected surface (known as the sliding surface or the sliding manifold [3]) in the system state space. A sliding surface should be designed to provide required system behavior. The system motion in sliding is governed by equations of sliding surface and closed-loop internal dynamics of the system if the total relative degree [4] of the system input/output dynamics is less than the order of the system. This motion is establishing as a result of steady auto-oscillations of very high frequency (infinite frequency in ideal sliding mode) induced in the closed-loop system that makes this motion to be robust to system 
parameter variations and external disturbances. The ideal system motion in sliding doesn't depend on control directly, a SMC provides for sliding surface to attract system trajectories and maintain them on the surface thereafter. As for high frequency switching of control vector components in auto-oscillation regime, those could be input voltages to the input of the actuator implementing actual control input to the system, if it's, say, a mechanical one. As far as an actuator is usually a low-pass filter, the actual control force will be a smooth continuous function of time.

The problem to attenuate the accelerations transmitted to an isolated experiment mount exhibits a non-minimum phase nature. As we'll see further, the relative position of the platform in the sway space is included in the system internal dynamics, which is unstable. That's why we'll use a Dynamic Sliding Manifold (DSM) technique [5] to address this non-minimum phase output stabilization problem. A sliding surface will be designed such that the platform acceleration dynamics behaves as a filter with specified characteristics with respect to input disturbances, and closed-loop internal dynamics are stable, which means that the relative position of the platform is bounded within specified limits. A high-order sliding mode on this surface will be used to provide existence of the sliding mode considering the actuator as uncertain dynamics of known order.

\section{Problem formulation}

A one-dimensional model of the system to be controlled presented in Fig. 1 can be described by the following equation

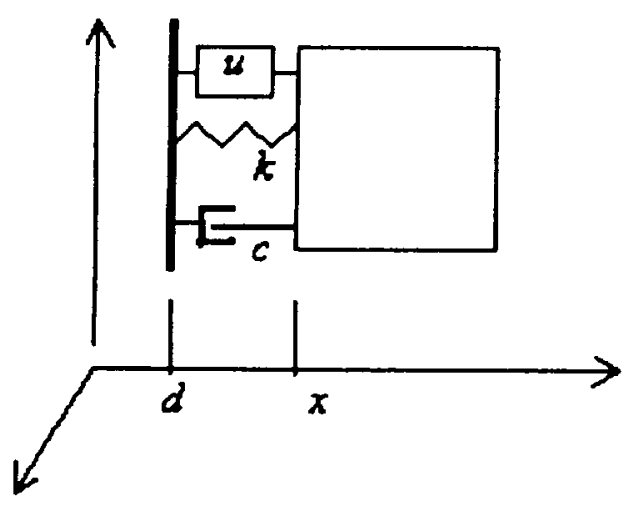

Fig. 1

$$
m \ddot{x}=-k(x-d)-c(\dot{x}-\dot{d})-u,
$$$$
\text { where }|d-x| \leq 0.01 \mathrm{~m},|u| \leq 4 N, m=10 \mathrm{~kg}, k=20 \frac{\mathrm{N}}{\mathrm{m}} \text {, }
$$

and $c=0.283$.

The system state vector is selected to be

$$
\left\{\begin{array}{l}
z_{1}=x-d, \\
z_{2}=s(x-d), \\
z_{3}=\frac{\omega_{h}}{s+\omega_{h}} s^{2} x, \omega_{h}=2 \pi \cdot 250 \mathrm{rad} / \mathrm{s}
\end{array}\right.
$$


in the Laplace domain, where $z_{3}$ is the output from the accelerometer. The state space equations of motion are shown as

$$
\left\{\begin{array}{l}
\dot{z}_{1}=z_{2} \\
\dot{z}_{2}=-\frac{k}{m} z_{1}-\frac{c}{m} z_{2}-\frac{1}{m} u-\ddot{d} \\
\dot{z}_{3}=-\frac{\omega_{h} k}{m} z_{1}-\frac{\omega_{h} c}{m} z_{2}-\omega_{h} z_{3}-\frac{\omega_{h}}{m} u
\end{array}\right.
$$

To transform the system (3) to the so-called normal form [4], we apply the following transformation

$$
\left[\begin{array}{l}
x_{1} \\
x_{2} \\
x_{3}
\end{array}\right]=\left[\begin{array}{ccc}
1 & 0 & 0 \\
0 & 1 & -\frac{1}{\omega_{h}} \\
0 & 0 & 1
\end{array}\right] \cdot\left[\begin{array}{l}
z_{1} \\
z_{2} \\
z_{3}
\end{array}\right] .
$$

The resulting system of equations represented in normal form is shown as

$$
\begin{cases}\dot{x}_{1}=x_{2}+\frac{1}{\omega_{h}} x_{3}, & \alpha=3141.59 \\ \dot{x}_{2}=x_{3}-\ddot{d}, & \beta=44.45 \\ \dot{x}_{3}=-c x_{1}-\beta x_{2}-\not x_{3}-b u, & \gamma=1570.83 \\ y=x_{3} & b=157.08\end{cases}
$$

The objective of our control effort is to force the output to zero $(y \rightarrow 0)$ and to maintain $\left|x_{1}(t)\right| \leq 0.01[\mathrm{~m}]$ for all time. This is a very challenging problem since the system (5) has unstable zero dynamics [3] with two eigenvalues at origin:

$$
\ddot{x_{1}}=-\ddot{d} \text {. }
$$

This is commonly characterized as a non-minimum phase system.

\section{SMC design}

It is well known [3] that a conventional sliding manifold cannot be used to stabilize $y \rightarrow 0$ for a non-minimum phase system under a bounded control law. However, using a dynamic sliding manifold (DSM) [5], we can achieve BIBO stability for the closed-loop system. We use a DSM of the form 


$$
\sigma=x_{3}+c_{1} \int x_{3} d \tau+c_{0} x_{1} .
$$

Assuming that the sliding mode exists in the DSM (6), we obtain, using (5) and (6), the acceleration dynamics in sliding mode as

$$
\ddot{x}_{3}+\left(c_{1}+\frac{c_{0}}{\omega_{h}}\right) \dot{x}_{3}+c_{0} x_{3}=c_{0} \ddot{d} .
$$

Also, the relative position dynamics (or closed-loop internal dynamics) is shown as

$$
\ddot{x}_{1}+\left(c_{1}+\frac{c_{0}}{\omega_{h}}\right) \dot{x}_{1}+c_{0} x_{1}=-\ddot{d}-c_{1} \dot{d} .
$$

Given that the acceleration $x_{3}$ and relative position $x_{1}$ are measurable, the sliding mode (6) can be achieved according to the following control law

$$
u=\hat{u}_{e q}+\frac{\rho}{b} \operatorname{sign}(\sigma), \rho>\left|\hat{u}_{e q}-u_{e q}\right|, \hat{u}_{e q}=-\frac{\alpha}{b} x_{1}+\frac{c_{1}-\gamma}{b} x_{3},
$$

where $u_{e q}$ is defined as the equivalent control [3].

Interpretation of the dynamic SMC as an active low-pass filter

The closed-loop acceleration dynamics (7) can be interpreted as a second order filter for $\ddot{d}$. This is written in the Laplace domain as

$$
x_{3}=\frac{c_{0}}{s^{2}+\left(c_{1}+\frac{c_{0}}{\omega_{h}}\right) s+c_{0}} \ddot{d} .
$$

Selecting $c_{0}=0.1, c_{1}=0.6$, we obtain amplitude characteristics for (10) presented in Fig. 2 .

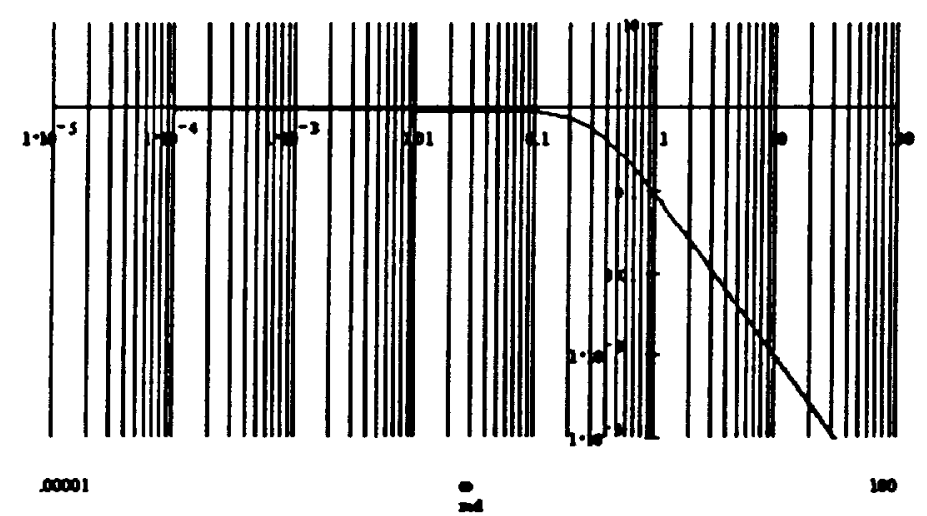

Fig. $2\left|\frac{x_{3}}{\ddot{d}}\right|$ versus $\omega[\mathrm{rad} / \mathrm{s}]$ 
Thus, in ideal sliding mode we've matched all the requirements for disturbance attenuation.

\section{SMC design for the system with second order uncertain actuator dynamics}

For the system shown in (5), a DSM (6) and a control law (9) have been designed to provide the existence of sliding mode such that closed-loop dynamics of the system (5) achieves the required performance (acceleration attenuation and bounded position deviation) assuming an ideal actuator. In reality, the control force $u$ is provided by an actuator system that can be modeled by a transfer function of the form

$$
u=\frac{\omega_{0}^{2}}{s^{2}+2 \varsigma \omega_{0} s+\omega_{0}^{2}} v, \omega_{0}^{2}=\hat{\omega}_{0}^{2}+\Delta \omega, \delta=\frac{\Delta \omega}{\hat{\omega}_{0}^{2}},|\delta|<1,
$$

or in differential equation format

$$
\left\{\begin{array}{l}
\dot{\eta}_{1}=\eta_{2}, \\
\dot{\eta}_{2}=-2 \varsigma \omega_{0} \eta_{2}-\omega_{0}^{2} \eta_{1}+\omega_{0}^{2} v, \\
u=\eta_{1},
\end{array}\right.
$$

where $v$ is the actual control input. Considering actuator (11) as uncertain second order system [6,7], we should provide existence of the sliding mode on the surface $\sigma=0$ using $v$ as control input. From (5),(6) we obtain

$$
\begin{aligned}
& \dot{\sigma}=-\alpha x_{1}+\left(c_{0}-\beta\right) x_{2}+\tilde{c} x_{3}-b u, \tilde{c}=\frac{c_{0}}{\omega_{n}}+c_{1}-\gamma, \\
& \ddot{\sigma}=-\alpha \widetilde{c} x_{1}-(\alpha+\beta \widetilde{c}) x_{2}-\left(\frac{\alpha}{\omega_{n}}+\beta-c_{0}+\gamma \tilde{c}\right) x_{3}-\left(c_{0}-\beta\right) \ddot{d}-b \widetilde{c} \eta_{1}-b \eta_{2},
\end{aligned}
$$

then, combining (12) and (13), we have

$$
\dddot{\sigma}=\varphi\left(x_{1}, x_{2}, x_{3}, \eta_{1}, \eta_{2}, t\right)-b \omega_{0}^{2} v,
$$

where $\varphi\left(x_{1}, x_{2}, x_{3}, \eta_{1}, \eta_{2}, t\right)=\widetilde{\alpha x_{1}}+\widetilde{\beta} x_{2}+\widetilde{\gamma} x_{3}+\widetilde{\delta} \eta_{1}+\tilde{\lambda} \eta_{2}+(\alpha+\beta \tilde{c}) \ddot{d}-\left(c_{0}-\beta\right) \ddot{d}$,

$\tilde{\alpha}=\alpha \tilde{\tilde{c}}, \tilde{\beta}=\beta \tilde{\bar{c}}-\alpha \tilde{c}, \tilde{\gamma}=\gamma \tilde{\tilde{c}}-(\alpha+\beta \tilde{c})-\frac{\alpha}{\omega_{n}} \tilde{c}, \tilde{\delta}=b \tilde{\tilde{c}}+b \omega_{0}^{2}, \tilde{\lambda}=-b \tilde{c}+2 b \varsigma \omega_{0}$,

$\widetilde{\widetilde{c}}=\frac{\alpha}{\omega_{n}}+\beta-c_{0}+\gamma \tilde{c}$

$\varphi(\cdot)$ is to be considered as uncertain but bounded term $\forall t$ in some domain in $\Re^{5}$. 
Thus, only the third derivative of sliding surface parameter $\sigma$ is proportional to control $\nu$. To provide convergence $\sigma$ to zero, we build an auxiliary DSM [8]

$$
J=\frac{s^{2}+b_{1} s+b_{0}}{s^{2}+a_{1} s+a_{0}} \sigma
$$

System motion in sliding manifold $J=0$ will provide $\sigma \rightarrow 0, \dot{\sigma} \rightarrow 0, \ddot{\sigma} \rightarrow 0$ according to

$$
\ddot{\sigma}+b_{1} \dot{\sigma}+b_{0} \sigma=0 \text {. }
$$

The sliding mode where $\sigma=\dot{\sigma}=\ddot{\sigma}=0$ is known as the third-order sliding mode or 3-sliding mode [9]. Thus, if we provide the asymptotic third-order sliding mode (16) that, in turn, will enforce given acceleration dynamics (10).

From equation (15) we obtain

$$
\dddot{J}+\left(a_{1}-b_{1}\right) \ddot{J}+\left(a_{0}-a_{1} b_{1}\right) \dot{J}-a_{0} b_{1} J=\left(b_{0}-b_{1}^{2}\right) \dot{\sigma}-b_{0} b_{1} \sigma+\dddot{\sigma} .
$$

If we select the control

$$
\begin{gathered}
\nu=\frac{\rho}{b \hat{\omega}_{0}^{2}} \operatorname{sgn}(J), \\
\rho=\frac{L+\tilde{\rho}}{1-\delta}, \tilde{\rho}>0, L=\max \left\{\frac{\left|\varphi(\cdot)+a_{0} b_{1} J+\left(b_{0}-b_{1}^{2}\right) \dot{\sigma}-b_{0} b_{1} \sigma\right|}{\mu}\right\}, \mu=\frac{1+\delta}{1-\delta}>1,(|\delta|<1),
\end{gathered}
$$

then from (14),(17),(18) we obtain the following closed-loop system

$$
\dddot{J}+\left(a_{1}-b_{1}\right) \ddot{J}+\left(a_{0}-a_{1} b_{1}\right) \dot{J}=\varphi(\cdot)+a_{0} b_{1} J+\left(b_{0}-b_{1}^{2}\right) \dot{\sigma}-b_{0} b_{1} \sigma-\gamma(L+\tilde{\rho}) \operatorname{sgn}(J) .
$$

The following proposition is valid in this case

Proposition Given conditions (19) for uncertain terms, $\exists \tilde{L}(\tilde{\rho})>0$ such that the solutions of the system (20) are bounded within domain $|J| \leqslant \frac{\tilde{L}}{\left(a_{1}-b_{1}\right)\left(a_{0}-a_{1} b_{1}\right)}, a_{1}-b_{1}>0, a_{0}-a_{1} b_{1}>0$ under control law (18).

Proof sketch: As soon as $\varphi(\cdot)+a_{0} b_{1} J+\left(b_{0}-b_{1}^{2}\right) \dot{\sigma}-b_{0} b_{1} \sigma$ is bounded in some domain of its arguments $\exists \infty>\tilde{L}>0$ such that $-\tilde{L} \operatorname{sgn}(J)$ will be the function majoring $\varphi(\cdot)+a_{0} b_{1} J+\left(b_{0}-b_{1}^{2}\right) \dot{\sigma}-b_{0} b_{1} \sigma-\gamma(L+\tilde{\rho}) \operatorname{sgn}(J)$. Using $-\widetilde{L} \operatorname{sgn}(J)$, we build equation for $\widetilde{J}$, which is majoring $J$ 


$$
\begin{aligned}
& \dddot{J}+\left(a_{1}-b_{1}\right) \ddot{\widetilde{J}}+\left(a_{0}-a_{1} b_{1}\right) \dot{\tilde{J}}+\tilde{L} \operatorname{sgn}(\widetilde{J})=0, \\
& \ddot{\widetilde{J}}+\left(a_{1}-b_{1}\right) \ddot{\widetilde{J}}+\left(a_{0}-a_{1} b_{1}\right) \dot{\tilde{J}}+\frac{\tilde{L}}{|\widetilde{J}|} \widetilde{J}=0 .
\end{aligned}
$$

The system (21) will be asymptotically stable if $a_{1}-b_{1}>0, a_{0}-a_{1} b_{1}>0 \frac{\widetilde{L}}{|\widetilde{J}|}>0$ and $\left(a_{1}-b_{1}\right)\left(a_{0}-a_{1} b_{1}\right)>\frac{\widetilde{L}}{|\widetilde{J}|}$. This fact means that we can select $a_{0}, a_{1}, b_{1}$ such that $\widetilde{J}$ will converge to the domain $|\widetilde{J}| \leq \frac{\tilde{L}}{\left(a_{1}-b_{1}\right)\left(a_{0}-a_{1} b_{1}\right)}$ only. Consequently, the same estimate we'll have for $/ /$. Selecting sufficiently large $\left(a_{1}-b_{1}\right)\left(a_{0}-a_{1} b_{1}\right)$, we can make this domain to be arbitrarily small.

\section{Simulations}

For the time simulations, we select the parameters $c_{0}=0.1, c_{1}=0.6$, $b_{1}=800, b_{0}=4 \cdot 10^{5}, a_{1}=4440, a_{0}=9.896 \cdot 10^{6}$, and $\tilde{\rho}=0.01$, and use a sliding mode controller of the form

$$
\sigma=x_{3}+c_{1} \int x_{3} d \tau+c_{0} x_{1}, J=\frac{s^{2}+b_{1} s+b_{0}}{s^{2}+a_{1} s+a_{0}} \sigma, v=-20 x_{1}-10 x_{3}+0.01 \operatorname{sgn}(J),
$$

for the system (5) with actuator (12), where according to (4) $x_{3}$ is the acceleration of the platform to be isolated, and $x_{1}$ is its relative position in sway space. The results of the simulations are given in Figs. 3-15. The external disturbance $\ddot{d}=0.01 \sin (2 \pi \cdot 10 t)\left[\mathrm{m} / \mathrm{s}^{2}\right]$ is presented in Fig. 3. The steady state value of the platform's acceleration is shown as $x_{3}=1 \cdot 10^{-5}\left[\mathrm{~m} / \mathrm{s}^{2}\right]$. The external disturbance has been attenuated by factor of 1000 , and the relative position has been held within prescribed limits (Fig.6). Fig.10 shows that the sliding mode is provided in the DSM $J$.

We evaluate robustness in the second test by changing the set of system parameters by $20 \%$ as follows: $k_{\text {new }}=k+0.2 k=24[\mathrm{~N} / \mathrm{m}], m_{\text {new }}=m-0.1 m=9[\mathrm{~kg}], c_{\text {new }}=c+0.2 c=0.3396$. This leads to a new natural frequency of the platform given by $\omega_{n_{m-n}}=1.633[\mathrm{rad} / \mathrm{s}]$. The second 
test has been carried out with the same external disturbance shown in Fig.3. From Fig. 12, we see that the desired disturbance attenuation (after transient is over) is achieved. Because of parameter variations, settling time of the closed-loop system increases from $20 s$ to $30 s$, but in steady state the system motion was insensitive to parameter.

\section{Conclusions}

The problem to attenuate the accelerations transmitted to an isolated experiment mount, which exhibits the nonminimum phase nature, has been solved using Dynamic Sliding Manifold technique. The platform closed-loop dynamics behaves as a filter with specified characteristics with respect to input vibrations. High-order sliding mode is achieved to be robust to uncertain second-order actuator dynamics via auxiliary DSM design.

\section{References}

(1) Grodsinsky, C. M. and Whorton, M. S., "A Survey of Active Vibration Isolation Systems for Microgravity Applications," Journal of Spacecraft and Rockets, submitted for publication.

(2) Knospe, C. R., Hampton, R. D., and Allaire, P. E., "Control Issues of Microgravity Vibration Isolation," Acta Astronautica, Vol. 25, No. 11, pp. 687-697, 1991.

(3) Utkin V. I., "Sliding Modes in Control Optimization", Springer, Berlin, 1992.

(4) Isidory A., "Nonlinear Control Systems", 3rd ed. London, Springer-Verlag, 1995.

(5) Shtessel Y.B., "Nonlinear nonminimum phase output tracking via dynamic sliding manifolds," J. Franklin Inst., Vol. 335B, No. 5, 1998, pp. 841-850.

(6) K.D. Young and P.V. Kokotovic, "Analysis of feedback loop interaction with parasitic actuators and sensors," Automatica, Vol. 18, pp.577-582, Sept., 1982.

(7) K.D. Young, V.I. Utkin, and Ü. Özgüner, “A Control Engineer's Guide to Sliding Mode Control," IEEE Transactions on Control Systems Technology, Vol. 7, No.3, 1999.

(8) D.Krupp, I.A.Shkolnikov and Y.B.Shtessel, "High Order Sliding Modes in Dynamic Sliding Manifolds: SMC Design with Uncertain Actuator," Proceedings of American Control Conference, 2000 - submitted.

(9) Levant A and L. Fridman, "High Order Sliding Modes as a Natural Phenomenon in Control Theory," in Robust Control via variable structure and Lyapunov techniques, F. Garofalo and L. Glielmo eds., Lecture Notes in Control and Information Science, No.217, pp.107-133, Springer-Verlag, London, 1996. 
Test 1

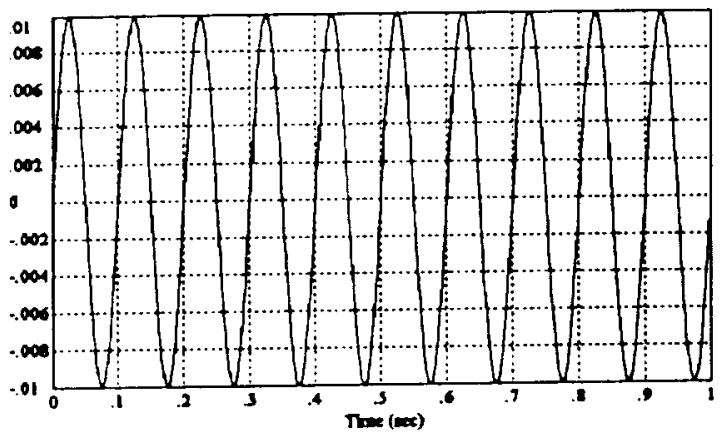

Fig. 3 Input disturbance $\ddot{d}\left[\frac{m}{s^{2}}\right]$ versus time for $1 \mathrm{sec}$

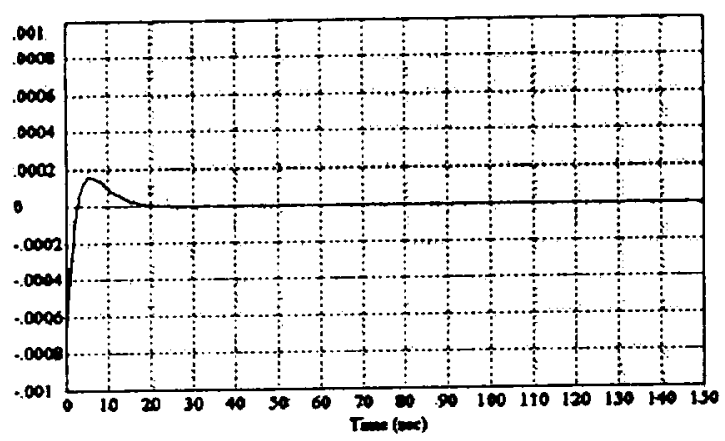

Fig.4 Acceleration $x_{3}\left[\frac{m}{s^{2}}\right]$ vs. time

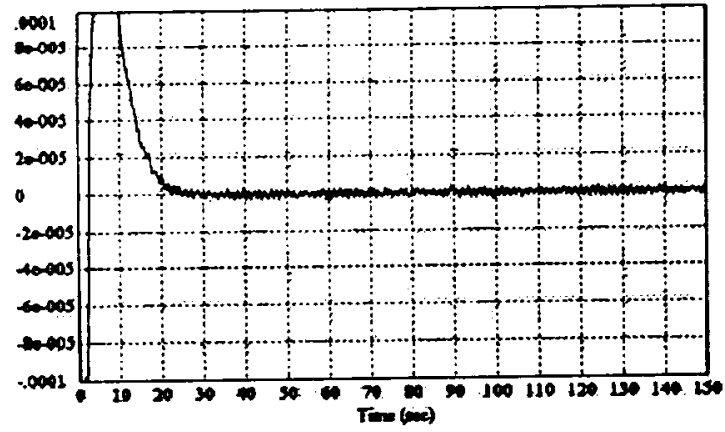

Fig.5 Acceleration $x_{3}\left[\frac{m}{s^{2}}\right]$ (high resolution)

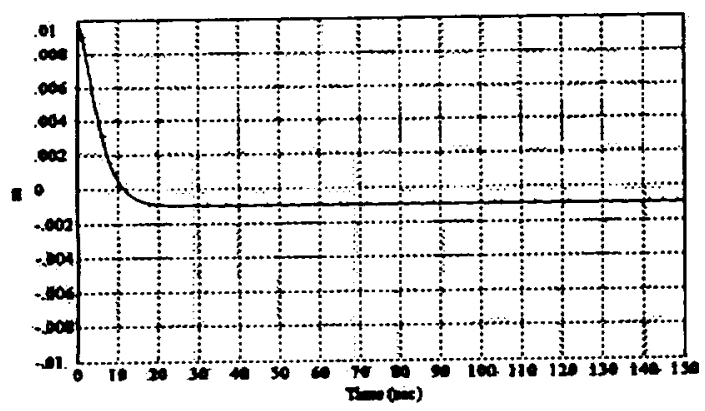

Fig.6 Relative position $x_{1}[m]$ versus time

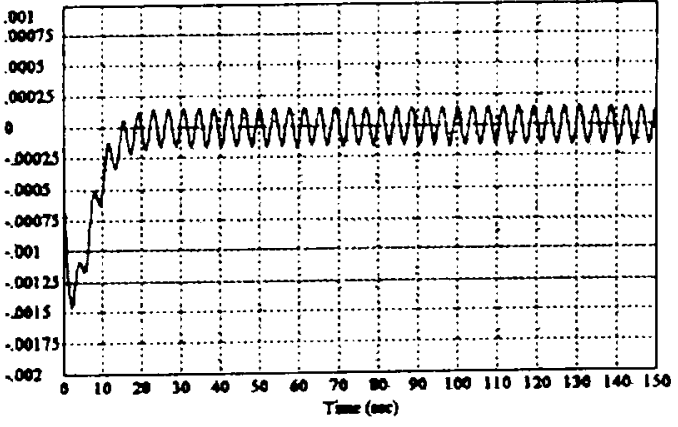

Fig.7 Second component of internal dynamics $x_{2}\left[\frac{m}{s}\right]$

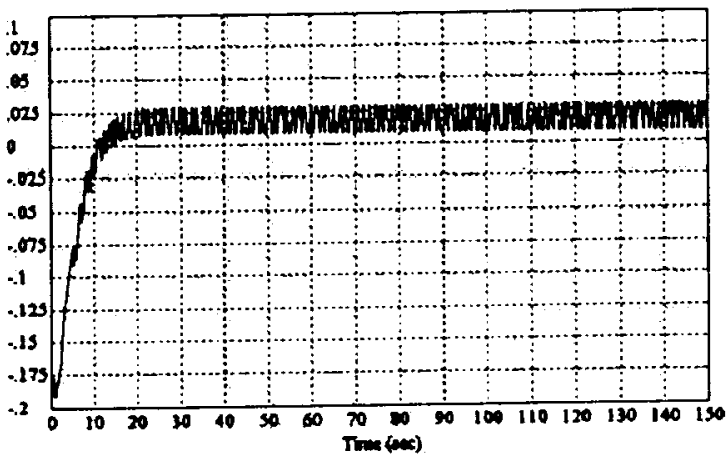

Fig. 8 Control input to the actuator $v$ vs. time

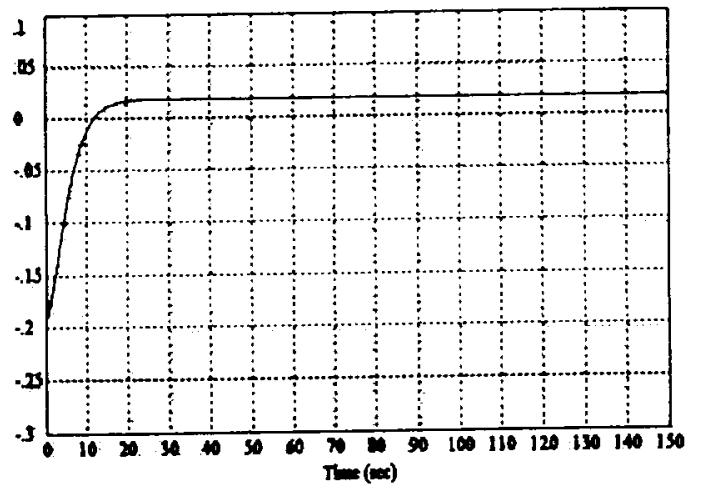

Fig.9 Control input to the plant $u[N]$ vs. time

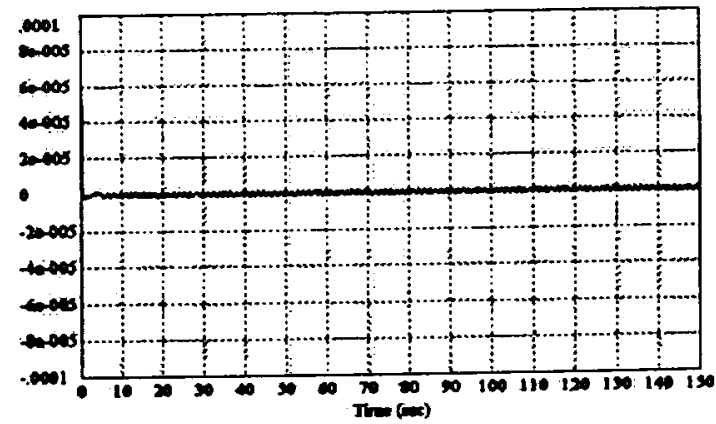

Fig.10 Auxiliary sliding surface $J$ vs. time 
Test 2

For new set of system parameters

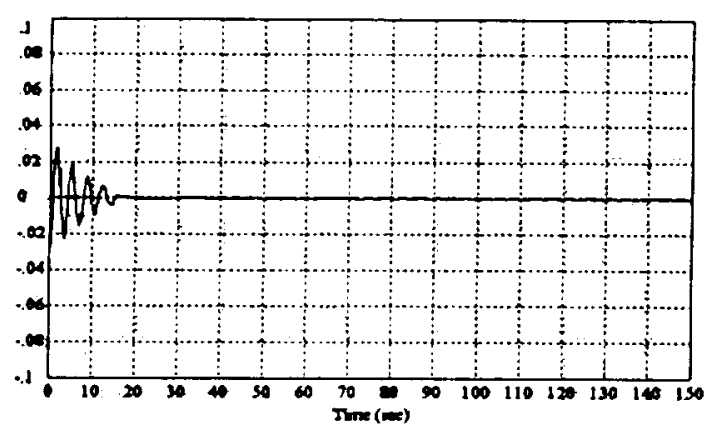

Fig.11 Acceleration $x_{3}\left[\frac{m}{s^{2}}\right]$ vs. time

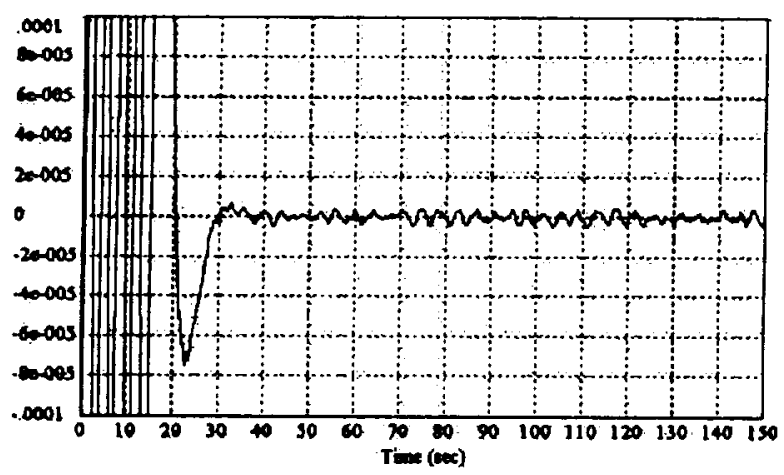

Fig.12 Acceleration $x_{3}\left[\frac{m}{s^{2}}\right]$ (high resolution)

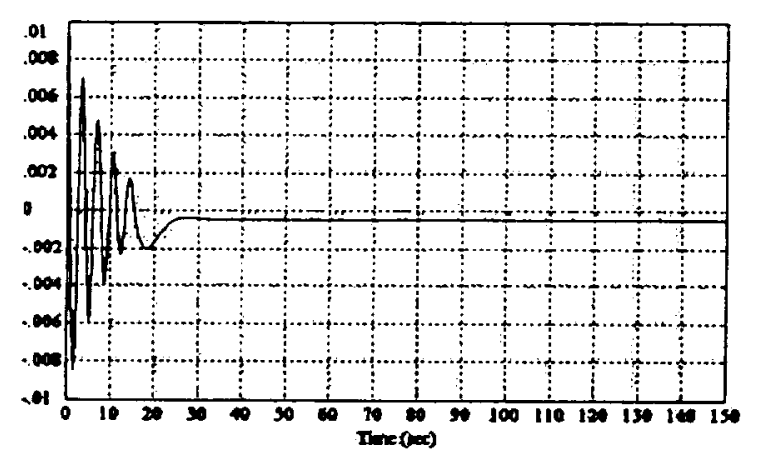

Fig.13 Relative position $x_{1}[m]$ vs. time

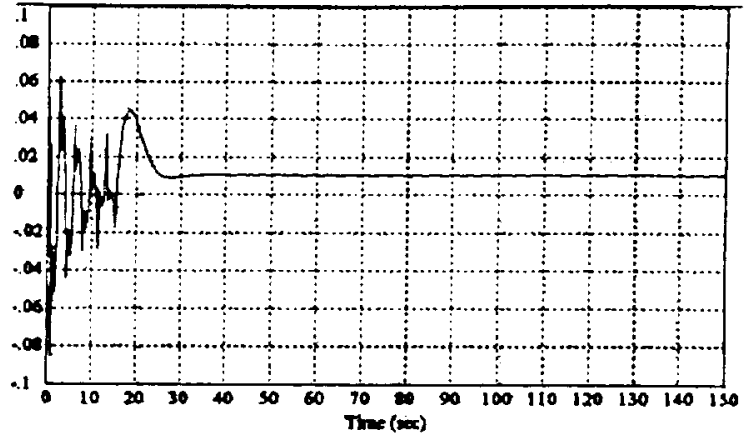

Fig. 14 Control input to the plant $u[N]$ vs. time

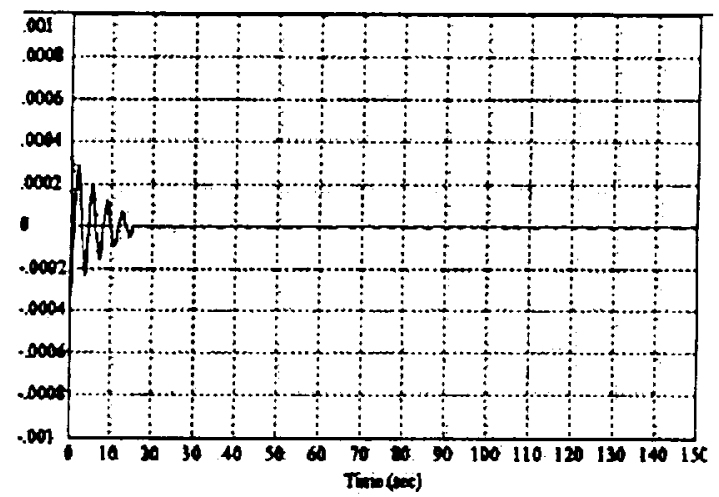

Fig. 15 Auxiliary sliding surface $J$ vs. time

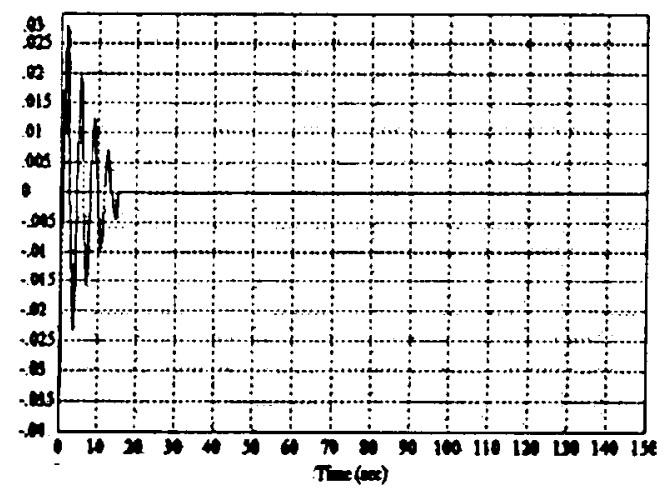

Fig. 16 Sliding surface $\sigma$ vs. time 